\title{
Generación de haz con momento angular orbital registrado en cristal fotorrefractivo
}

Generation of a beam with orbital angular momentum recorded on the photorefractive crystal

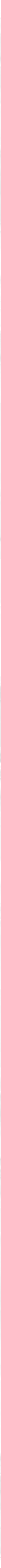




\title{
Generación de haz con momento angular orbital registrado en cristal fotorrefractivo ${ }^{1}$
}

\section{Generation of a beam with orbital angular momentum recorded on the photorefractive crystal}

\author{
Sandra J. M. Carreño ${ }^{2}$, André L. Moura ${ }^{3}$, Vladimir Jerez ${ }^{4}$ \\ ${ }^{2}$ Departamento de Física, Universidade Federal de Pernambuco - UFPE, Recife, Brazil \\ ${ }^{3}$ Núcleo de Ciências Exatas, Universidade Federal de Alagoas, Arapiraca, Brazil \\ ${ }^{4}$ Grupo de Investigación FIELDS, Universitaria de Investigación y Desarrollo
}

Artículo recibido en julio de 2016; artículo aceptado en agosto de 2016 - UDI

Citación del artículo: Carreño, S., Moura, A. \& Jerez, V. (2017). Generación de haz con momento angular orbital registrado en cristal fotorrefractivo. I+D Revista de Investigaciones, 9 (1), 146 - 149

\begin{abstract}
Resumen
En este trabajo se presenta una técnica novedosa para la generación de haces de luz con momento angular orbital (MAO), utilizando un cristal fotorrefractivo Bi12TiO20 (BTO) en un arreglo experimental donde un haz con MAO y un haz de referencia generan un registro holográfico interferométrico del haz. Una de las posibles aplicaciones, si se consigue fijar el holograma, podría ser el estudio de fenómenos de óptica y óptica no lineal utilizando haces con altas potencias.
\end{abstract}

Palabras clave: Momento angular orbital, cristal fotorrefractivo, BTO.

\begin{abstract}
This work presents a novel technique for the generation of light beams with orbital angular momentum (MAO) using a photorefractive BTO crystal in an experimental arrangement where a beam with $\mathrm{MAO}$ and a reference beam generate a holographic interferometric beam register. One of the possible applications if you can fix the hologram, could be the study of phenomena of optics and non-linear optics using beams with high powers.
\end{abstract}

Key words: Orbital angular momentum, photorefractive crystal, BTO.

1. Artículo de tipo experimental, enfoque cualitativo, resultado de un proyecto de investigación (culminado), perteneciente al área de Física, subárea de Óptica, desarrollado en el grupo de investigación Fields, financiado por la Universitaria de Investigación y Desarrollo de la ciudad de Bucaramanga (Colombia). Dirección Calle 9 n. 23-55, PBX: 6352525. Fecha de inicio: febrero de 2016, fecha de terminación: diciembre de 2016. 2. Magíster en Física, Universidad Federal de Pernambuco (UFPE). Docente- investigador del grupo Óptica No Lineal Universidad UFPE de la ciudad de Recife (Brasil): Dirección: Av. Prof. Moraes Rego, 1235, PBX: +55 81 2126-8000. Correo institucional: s.carreno@df.ufpe.br

3. Físico de la Universidad Federal de Alagoas (UFAL). Doctor en Física UFAL. Docente- investigador del grupo Óptica de la UFAL de la ciudad de Arapiraca (Brasil): Dirección: Av. Manoel Severino, PBX: 5582 3214-1100. Correo institucional: andre.moura@fis.ufal.br

4. Físico de la Universidad Industrial de Santander (Bucaramanga). Doctor Física Universidad Industrial de Santander. Docente- investigador del grupo Fields, Universitaria de Investigación y Desarrollo de la ciudad de Bucaramanga (Colombia): Dirección: Calle 9 n. ${ }^{\circ} 23-55$ PBX 6352525. Correo institucional: vjerez2@udi.edu.co 
En esta investigación se buscó un método alternativo para generar haces con MAO. Las técnicas usadas hasta el momento para generar haces son los hologramas en forma de tenedor (Bazhenov et al., 1990) y la placa de espiral de fase (García et al., 2009); asimismo, para generar MAO también se pueden convertir modos Hermítico-Gaussianos en modos Laguerre-Gaussianos usando un sistema astigmático con dos lentes. (Allen, Beijersbergen, Spreeuw \& Woerdman, 1992)

En esta investigación se consiguió un método novedoso para generar haces con MAO utilizando el cristal fotorrefractivo Bi12TiO20 (BTO), materiales que son muy estudiados por su capacidad de registrar hologramas y sus aplicaciones, ampliamente conocidas (Frejlich, 2007; Günter, 1988). En esta investigación se aporta una nueva aplicación de estos materiales, utilizados para la generación de un haz con MAO.

Para el estudio de las propiedades ópticas de la materia y su interacción con haces con momento angular con bajas y altas potencias se hace necesario la creación de fuentes de haces con MAO con potencias altas, puesto que los sistemas actuales de generación de estos haces generan altas pérdidas y la energía que soportan es limitada, es el caso de experimentos como el de láser aleatorio bombeado con haces MAO altas potencias, el cual hasta el momento no se ha podido realizar. (Anderson et al., 2015; Maia et al., 2015)

El interés académico se basa en los estudios anteriores realizados con cristales fotorrefractivos, en los cuales se consiguió fijar hologramas en cristales de Niobatio de Litio, los cuales soportan haces de alta potencia; además, las pérdidas registradas para estos hologramas son bajas, teniendo una eficiencia de difracción del 95\% (Jerez, de Oliveira \& Frejlich, 2009). Con el interés de fijar un holograma con $\mathrm{MAO}$, como un primer paso, se consiguió registrar un haz con MAO; el paso siguiente para una próxima investigación es el fijado del holograma utilizando cambios térmicos y autoestabilización. Teniendo en cuenta que el cristal utilizado en este experimento BTO ya reportó la posibilidad del fijado del holograma (Jerez, de Oliveira, Frejlich, 2009).

\section{Experimento}

El registro se consigue con un arreglo experimental muy sencillo: la luz láser de longitud de onda de $435 \mathrm{~nm}$ con polarización vertical entra en un filtro espacial donde se colima la luz; este haz es partido en dos en el divisor de haz (DH). El haz de lectura se dirige al espejo M2 para incidir en el cristal de BTO en un ángulo de $45^{\circ}$; el haz de registro se dirige a la máscara de fase usada para generar el haz con momento angular con carga topológica +2 (modulador espacial de luz, Holoeye, LC-2002), luego los dos haces interfieren en el cristal BTO después de recorrer los trayectos abc y aec, de igual longitud. El tiempo de registro de los hologramas fue de un minuto, tiempo necesario para tener un holograma de volumen en el cristal BTO. Este experimento se realizó en una mesa de laboratorio aislada de las vibraciones y de luz externa; el arreglo experimental se muestra en la Figura 1. Para leer el holograma se bloqueó el haz de registro; por su parte, el haz de lectura incide sobre cristal y el holograma trasmitido es registrado en la cámara CCD (Edmund Optics, Model 1312c).

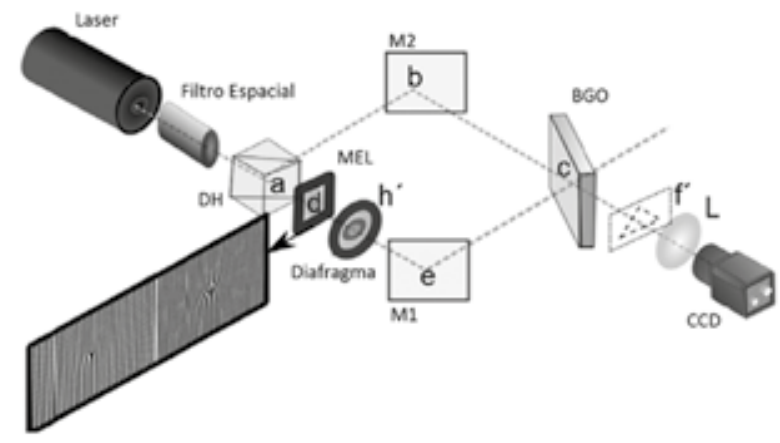

Figura 1.

Arreglo experimental

\section{Resultados y discusión}

La Figura 2 muestra la distribución de intensidad del interferograma registrado en el cristal BTO con el haz de lectura en el plano focal de la lente, para una carga topológica de 2 positiva de un holograma de tenedor generado por computador. Para verificar que el holograma corresponde a un haz con momento angular orbital, se usó una apertura triangular en una posición f' dentro del experimento mostrado en la Figura 1.
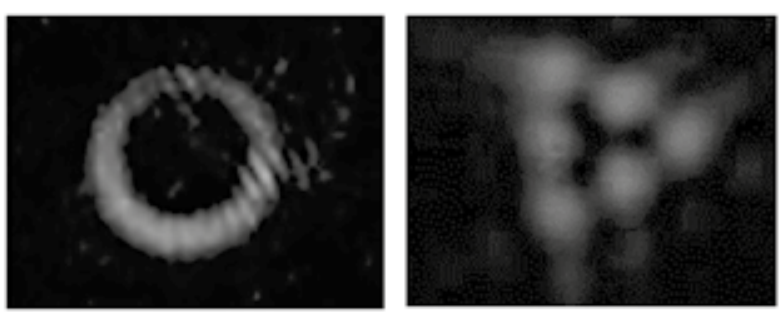

Figura 2.

Haz difractado con MAO por el cristal BTO y patrón de difracción de Fraunhofer 
La imagen a la izquierda de la Figura 2 muestra el patrón de difracción de campo lejano para una apertura triangular de un haz con momento angular orbital y carga topológica 2; la lente está en el plano focal de la dona registrada en el cristal BTO. Se pudo observar que el patrón de difracción se ajusta muy bien con los resultados de la difracción de Fraunhofer de un IOAM (Hickmann, Fonseca, Soares \& Chávez, 2010).

\section{Referencias}

Allen, L., Beijersbergen, M., Spreeuw, R. \& Woerdman, J. (1992). Orbital angular momentum of light and transformation of Laguerre-Gauus laser modes. Physical Review, 45. doi. org/10.1103/PhysRevA.45.8185

Anderson, S., Gomes, L., Raposo, E., Moura, A., Serge, I., Fewo, De Araújo, C. (2015). Random lasers, L'evy statistics and spin glasses: Synergy between photonics and complex systems.

Bazhenov, V. Y., Vasnetsov, M. V., \& Soskin, M. S. (1990). Laser beams with screw dislocations in their wavefronts. American Institute of Physics, 52(8), 429-431. doi:195.178.214.34/ps/1159/.

Frejlich, J. (2007). Photorefractive materials: Fundamental concepts, holographic recording and materials characterization (cap. 2). New Jersey: John Wiley \&Sons.
García, H. \$ Gutiérrez-Vega L.C. (2009). Diffraction of wave planes by fnite-radius spiral phase wave plates of integer and fractional topological charge. Journal Optical Society of America, 26(4), 794-803.

Günter, P. (1988). Photorefractive Effects and Materials, Topics in Applied Physics: Photorefractive Materials and Their Applications I and II (61-62). Günter, P. \& Huignard, J. (Eds). Berlín, Heidelberg: Springer-Verlag.

Hickmann, J., Fonseca, E., Soares, W. \& Chávez, S. (2010). Unveiling a truncated optical lattice associated with a triangular aperture using. Physical Review Letters, 105.

Jerez, V., de Oliveira, I. \& Frejlich, J. (2011). Fixed photorefractive holograms with maximum index-of-refraction modulation in LiNbO3:Fe. Journal of Applied Physics, 106 (6). doi. org/10.1063/1.3223319

Jerez, V., de Oliveira, I. \& Frejlich. J. (2009). Optical recording mechanisms in undoped titanosillenite crystals. Journal of applied physics, 109 (2). doi.org/10.1063/1.3533421

Maia, L., Filho, F., Jerez, V., Moura, A. \& de Araújo, C. (2015). Structural and luminescence properties of $\mathrm{Nd} 3+/ \mathrm{Yb} 3+$ codoped Al4B2O9 nanocrystalline powders. Journal of Materials Chemistry C, 3 (44). doi:10.1039/C5TC01696G 